\title{
Binder saturation, layer thickness, drying time and their effects on dimensional tolerance and density of cobalt chrome- tricalcium phosphate biocomposite
}

\author{
John Ruprecht, Kuldeep Agarwal and Shaheen Ahmed \\ Department of Automotive and Manufacturing Engineering Technology, Minnesota State \\ University, Mankato, 56001, US
}

\begin{abstract}
$\underline{\text { Abstract }}$
Traditional metals such as stainless steel, titanium and cobalt chrome are used in biomedical applications (implants, scaffolds etc.) but suffer from issues such as osseointegration and compatibility with existing bone. One way to improve traditional biomaterials is to incorporate ceramics with these metals so that their mechanical properties can be similar to cortical bones. Tricalcium phosphate is such a ceramic with properties so that it can be used in human body. This research explores the use of binder jetting based additive manufacturing process to create a novel biocomposite made of cobalt chrome and tricalcium phosphate. Experiments were conducted and processing parameters were varied to study their effect on the printing of this biocomposite. Layer thickness, binder saturation and drying time affected the dimensional tolerance and the density of the green samples. This effect is important to understand so that the material can be optimized for use in specific applications.
\end{abstract}

\section{Keywords:}

Additive Manufacturing, Binder Jetting.

\section{Introduction}

Biomaterial is any material, natural or man-made, that can perform a body function or can replace a body part or tissue. Depending on their application, biomaterials can be made from polymers, metals, ceramics or composites [1-3].

Biomedical implants can be classified into 3 major categories: External to the body (nonclinical, which includes surgical instruments, prosthetics etc.), Internal to the body \& permanent (includes hip implants, knee implants, stents etc.) and Internal to the body \& temporary (includes scaffolds, degradable screws and drug delivery systems) [1-3,14].

According to FDA, a "permanently implantable device is a device that is intended to be placed into a surgically or naturally formed cavity of the human body for more than one year to continuously assist, restore, or replace the function of an organ system or structure of the human body throughout the useful life of the device." Some examples include knee and hip implants. Temporary implants are commonly used in sports surgeries, such as in shoulder and knee ligamentous reconstruction and spinal reconstructive surgery [5, 6, 14]. 
The most common biomaterials for implants are metals and alloys, ceramics and polymers. In the past few years, Cobalt alloys have gained popularity for being used as biomaterials for implants. Bones have elastic moduli 7 -30 GPa, yield stress 30-70 MPa, compressive strength 100$230 \mathrm{MPa}$, and tensile strength $70-150 \mathrm{MPa}$. First generation of implants focused on replacement of the bone with a metal implant. However, mechanical properties of metals differ considerably from natural bone: Cobalt chrome alloys elastic modulus $=210 \mathrm{GPa}$, yield strength $=120-600$ $\mathrm{MPa}$, tensile strength 190 - $800 \mathrm{MPa}[3,4,7]$.

These differences lead to stress shielding resulting in loosening of the implant due to degradation of human tissues around implants and, consequently, further surgeries to replace the implants. Ceramics are inorganic materials with high compressive strength and biological inertness that make them suitable for scaffolds used in strengthening or replacing damaged bones and tissues. The most commonly used bioceramics are metallic oxides (e.g., $\mathrm{Al}_{2} \mathrm{O}_{3}, \mathrm{MgO}$ ), calcium phosphate (e.g., hydroxyapatite (HA), tricalcium phosphate (TCP), and octacalcium phosphate (OCP)), and glass ceramics (e.g. Bioglass, Ceravital).

Calcium phosphates have the best biocompatibility and properties closest to natural bones: elastic modulus 7-13 GPa, compressive strength 350-450 MPa, tensile strength 38-48 MPa and flexural strength 100-120 MPa. However, they have poor fracture toughness and tensile strength that limits their application to bioimplants. Several in vitro and vivo works have shown that calcium phosphates support the adhesion, differentiation and proliferation of osseogenesis-related cells (e.g., osteoblasts, mesenchymal stem cells), besides inducing gene expression in bone cells. The most important calcium phosphate is hydroxyapatite $\left(\mathrm{HA}, \mathrm{Ca}_{10}\left(\mathrm{PO}_{4}\right)_{6}(\mathrm{OH})_{2}\right)$ with chemical characteristics similar to hard tissues such as bone and teeth, that promotes hard tissue ingrowth and osseointegration when implanted into the human body. The porous structure of this material can be tailored to suit the interfacial surfaces of the implant. As a bulk material, HA lacks sufficient tensile strength and is too brittle to be used in most load bearing applications. In such cases, HA is coated onto a metal core or incorporated into polymers as composites. The ceramic coating on the titanium implants improves the surface bioactivity but often fails as a result of poor ceramic/metal interface bonding [8-11].

a-TCP and B-TCP are the two crystalline varieties of HA of interest in biological applications. $\mathrm{B}$-TCP is the thermodynamically stable form at low temperature. It transforms into $a$-TCP in the temperature range $1120-1170{ }^{\circ} \mathrm{C}$. B-TCP is generally preferred in sintered ceramic implants, while a-TCP is more commonly used in bone graft cements because of its hydrolysis properties. The requirements that allow bone ingrowth are a porosity of 30-70 vol \% and a pore diameter between 300 and $800 \mu \mathrm{m}$, mechanical properties of $0.5-15 \mathrm{MPa}$ similar to cancellous bone.

Thus there is a drive in the biomedical industry to create novel materials that behave very similar to bone and can be used for multiple applications: from permanent to temporary implants. Furthermore, these materials need to be manufactured in a manner that would create porosity in situ for biological applications.

Due to the versatility of additive manufacturing, it is gaining a lot of popularity in the field of bone implants. Selective Laser Sintering, Selective Laser Melting, Electron Beam Melting and Binder jet manufacturing have all been used to create various porous structures for biomedical implants [7, 9]. To accomplish the various different requirements of the implants it is necessary to create biocomposites that can have the strength properties of metals as well as the biological 
properties of bioceramics.

This research explores the use of binder jetting based additive manufacturing process to create a novel biocomposite made of cobalt chrome (CC) and tricalcium phosphate (TCP). Experiments were conducted and processing parameters were varied to study their effect on the printing of this biocomposite. Layer thickness, binder saturation and drying time affected the dimensional tolerance and the density of the green samples. This effect is important to understand so that the material can be optimized for use in specific applications.

\section{Binder.Jet-based Additive Manufacturing}

Binder Jetting AM is a Drop on Demand (DoD) inkjet printing process in which binder is emitted through a nozzle to form a short jet. This jet condenses into a drop and the position at which each drop lands on substrate is controlled by relative motion between drop and the substrate. The nozzle head is piezoelectric and uses the deformation of ceramic element to generate a pressure pulse needed to eject the binder. Typical drop diameters vary from $10 \mu \mathrm{m}-100 \mu \mathrm{m}$, drop volumes vary from $0.5-500 \mathrm{pl}$ and the drop speeds are $5-8 \mathrm{~m} / \mathrm{s}$ [15].

The main technique of manufacture using the Binder Jet process is as follows: (a) The CAD file is sliced into layers and a STL file is generated, (b) Each layer begins with a thin distribution of powder spread over the surface of a powder bed, (c) Using a technology similar to ink-jet printing, a binder material selectively joins particles where the object is to be formed, (d) A piston that supports the powder bed and the part-in-progress lowers so that the next powder layer can be spread and selectively joined, (e) This layer-by-layer process repeats until the part is completed, (f) Following a heat treatment, unbound powder is removed and the metal powder is sintered together. Fig. 1 shows the details of the whole process.

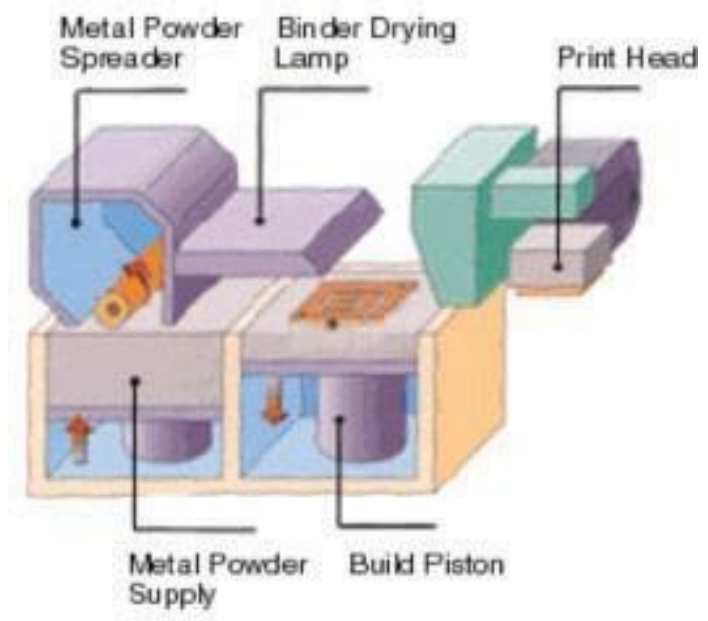

Fig. 1: Schematic of the Binder Jet Process (Courtesy The ExOne Company)

\section{Process parameters}

The Binder Jet process described above can be divided into 3 basic steps: 1) Binding, 2) Curing and 3) Sintering. There are various process parameters that can be changed to obtain a customized part in each of these steps. These include powder size, layer thickness during binding, part 
orientation in bed, heater power, roller speed, curing temperature, curing time, sintering time, sintering temperature, and sintering atmosphere. In this research we concentrated on the feasibility of printing of the CC and TCP biocomposite.

\section{Experimental Plan}

The materials used in the study were cobalt chrome (Co 212-H, Sandvik Osprey), which has a mean particle size of $53 \mu \mathrm{m}$ and an apparent density of $3.15 \mathrm{~g} / \mathrm{cc}$. The chemical composition of Co $212-\mathrm{H}$ is shown in Table 1.

\begin{tabular}{|l|l|l|l|l|l|l|l|}
\hline $\mathrm{C}$ & $\mathrm{Ni}$ & $\mathrm{Fe}$ & $\mathrm{Si}$ & $\mathrm{Mn}$ & Mo & $\mathrm{Cr}$ & Co \\
\hline $0.02 \max$ & $0.10 \max$ & $\begin{array}{l}0.75 \\
\max \end{array}$ & $1.00 \max$ & $\begin{array}{l}1.00 \\
\max \end{array}$ & $5.0-7.0$ & $27.0-30.0$ & Balance \\
\hline
\end{tabular}

Table 1: Chemical composition of Co 212-H (wt \%)

B-Tricalcium Phosphate (TCP) was obtained from Sigma-Aldrich (21218) with a mean particle size of $5 \mu \mathrm{m}$ and an apparent density of $1.92 \mathrm{glcc}$. Scanning Electron Microscope (JEOL JSM-6510MV) was used to look at the sizes and distribution of the powders before the process. The images are shown in Fig. 2.

Co 212-H was used as a benchmark and is called Sample Set 1. The two powders were mixed in with $80 \%$ Co 212-H -20\% TCP volume fraction and this is called sample set 2 .
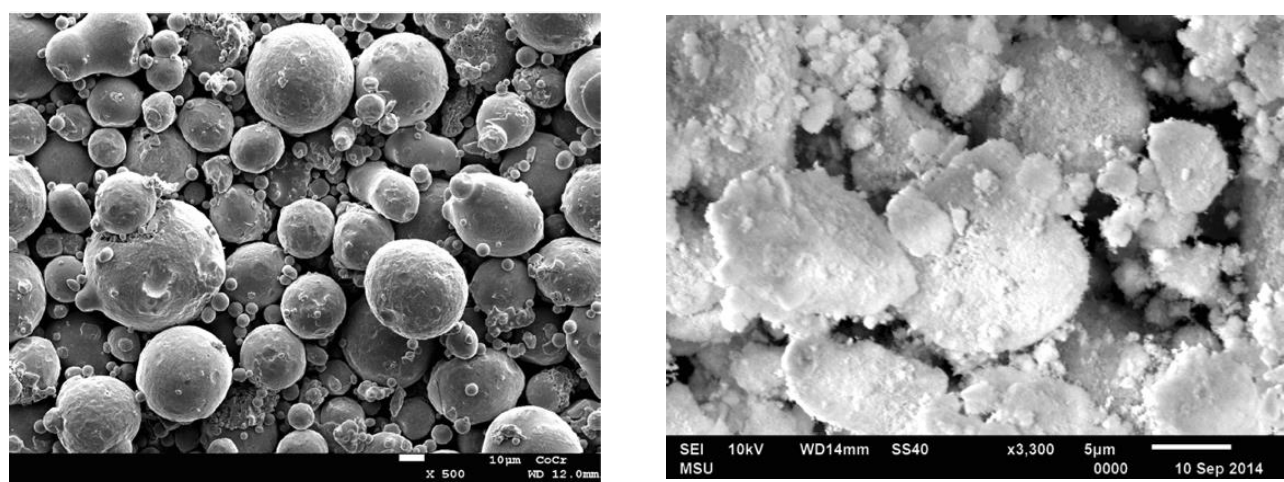

Fig. 2: SEM Micrographs for Co 212-H and TCP

The 2 sample sets were then used as the input powders in the binder jet additive manufacturing. To understand the effect of printing parameters of the binder jet process on the print quality, multiple factors and levels were chosen based on prior experience (Table 2). After the printing, all the samples were cured at $175^{\circ} \mathrm{C}$ for 3 hours.

The print quality was measured by 2 means: dimensional tolerance compared to the CAD model and the density of the part after the printing. Two different size of parts were printed to study the effect of size on the dimensional tolerance. The CAD drawings of the parts with nominal dimensions is shown in Fig. 3. Eight parts of $10 \mathrm{~mm}$ and 3 parts of $30 \mathrm{~mm}$ were printed for the corresponding experimental run. 


\begin{tabular}{|c|c|c|}
\hline S.No. & Factor & Levels \\
\hline 1 & Layer Thickness & $60 \mu \mathrm{m}, 90 \mu \mathrm{m}$ \\
\hline 2 & Binder Saturation (as \% of printhead max) & 80,100 \\
\hline 3 & Drying Time between layers & $15 \mathrm{sec}, 20 \mathrm{sec}$ \\
\hline 4 & Sample Size (Outside dimension) & $10 \mathrm{~mm}, 30 \mathrm{~mm}$ \\
\hline
\end{tabular}

Table 2: Factors and levels for Design of experiments

If a full factorial experimental design would be done on these parameters, it would require a total of 16 experiments. Instead a half factorial experimental design was chosen and 8 experiments were conducted. The experimental design matrix is shown in Table 3.
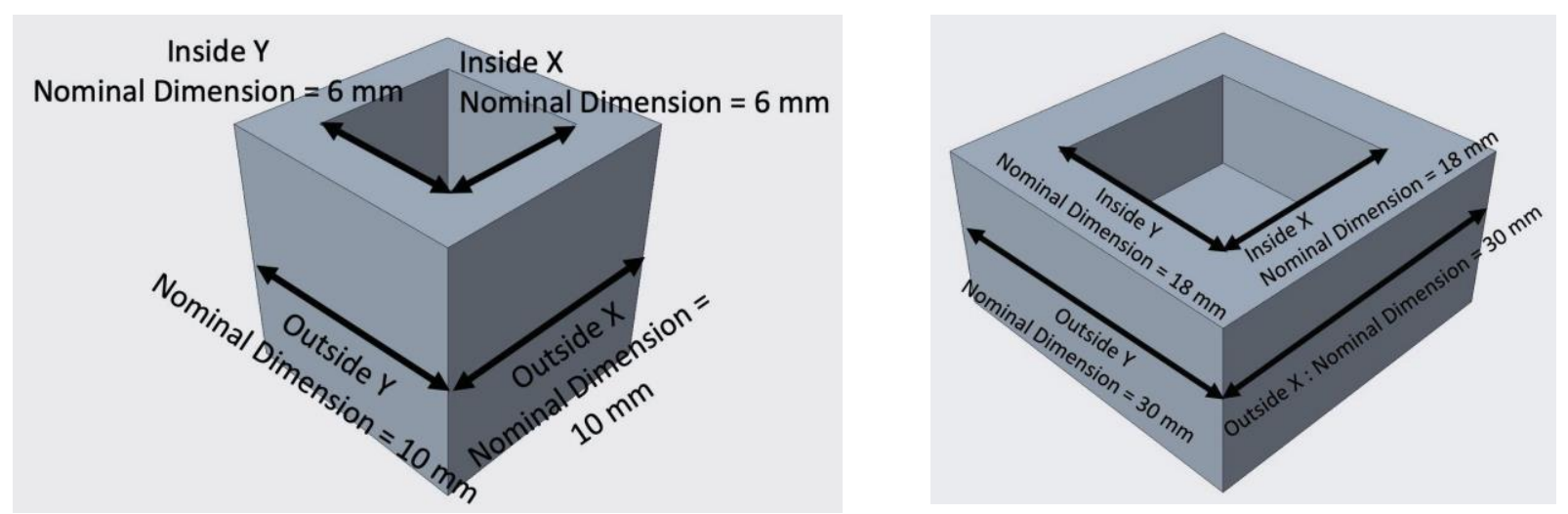

Fig. 3: CAD models with dimensions for $10 \mathrm{~mm}$ and $30 \mathrm{~mm}$ samples

\begin{tabular}{|c|c|c|c|c|}
\hline Experiment No. & Layer Thickness & Binder Saturation & Drying Time & Sample Size \\
\hline 1 & $90 \mu \mathrm{m}$ & $100 \%$ & $15 \mathrm{sec}$ & $10 \mathrm{~mm}$ \\
\hline 2 & $60 \mu \mathrm{m}$ & $100 \%$ & $20 \mathrm{sec}$ & $10 \mathrm{~mm}$ \\
\hline 3 & $90 \mu \mathrm{m}$ & $100 \%$ & $20 \mathrm{sec}$ & $30 \mathrm{~mm}$ \\
\hline 4 & $90 \mu \mathrm{m}$ & $80 \%$ & $20 \mathrm{sec}$ & $10 \mathrm{~mm}$ \\
\hline 5 & $60 \mu \mathrm{m}$ & $80 \%$ & $15 \mathrm{sec}$ & $10 \mathrm{~mm}$ \\
\hline 6 & $90 \mu \mathrm{m}$ & $80 \%$ & $15 \mathrm{sec}$ & $30 \mathrm{~mm}$ \\
\hline 7 & $60 \mu \mathrm{m}$ & $100 \%$ & $15 \mathrm{sec}$ & $30 \mathrm{~mm}$ \\
\hline 8 & $60 \mu \mathrm{m}$ & $80 \%$ & $20 \mathrm{sec}$ & $30 \mathrm{~mm}$ \\
\hline
\end{tabular}

Table 3: Experimental design matrix

\section{$\underline{\text { Results }}$}

After the parts were printed, they were weighed and measured for the outside $\mathrm{X}$, outside $\mathrm{Y}$, inside $\mathrm{X}$ and inside $\mathrm{Y}$ dimensions. Dimensional deviation from the $\mathrm{CAD}$ model was calculated according to the following formula for each of the 4 dimensions:

Dimensional Deviation $(\%)=\frac{\text { Nominal Dimension }(C A D)-\text { Measured Dimension }}{\text { Nominal Dimension }(C A D)} * 100$ 
Fig. $4-6$.

The box plots for the 2 different sample sets and their dimensional variations are shown in
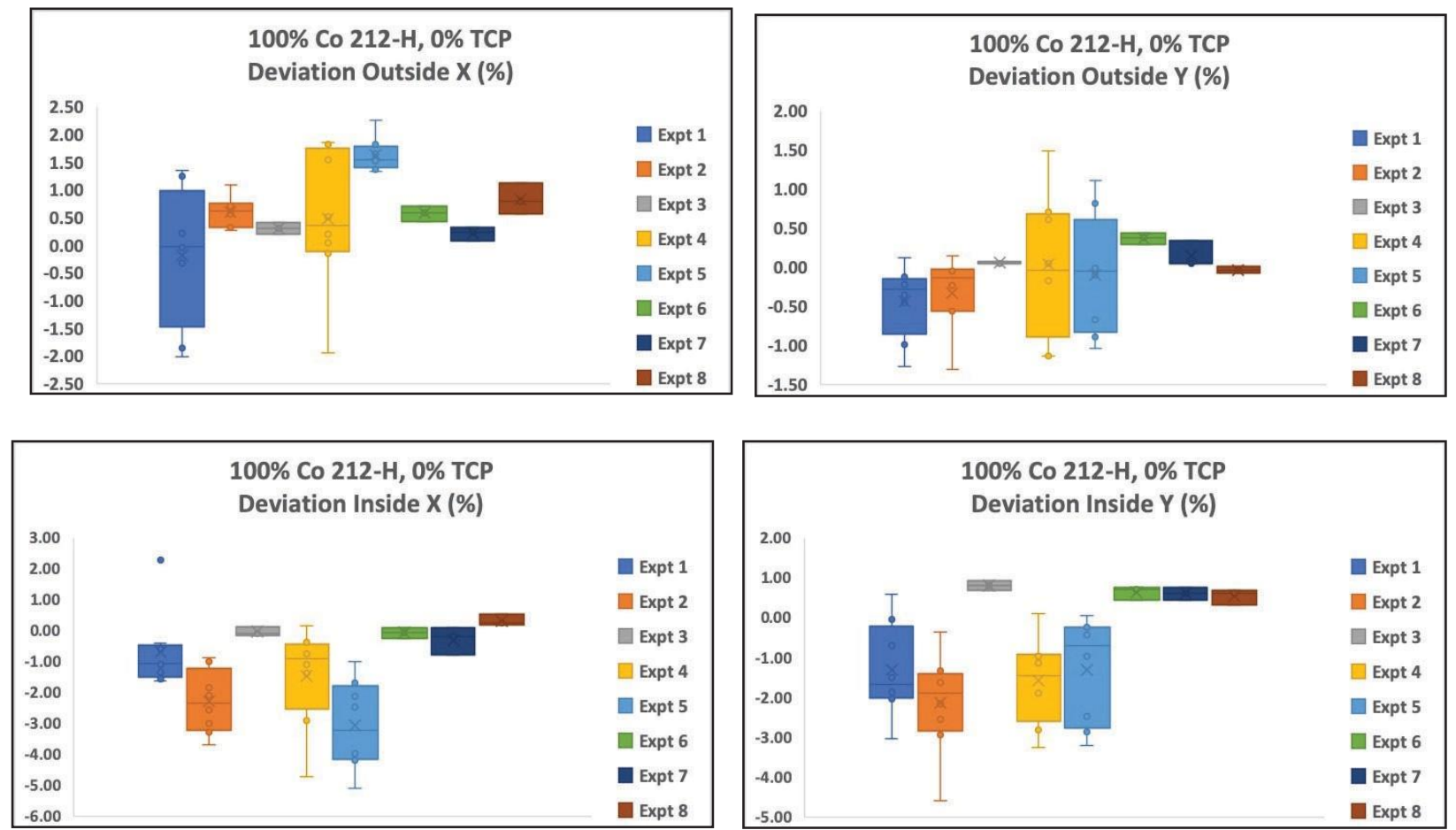

Fig. 4: Dimensional variation along 2 directions for sample set 1 (100\% Co 212-H, $0 \%$ TCP)

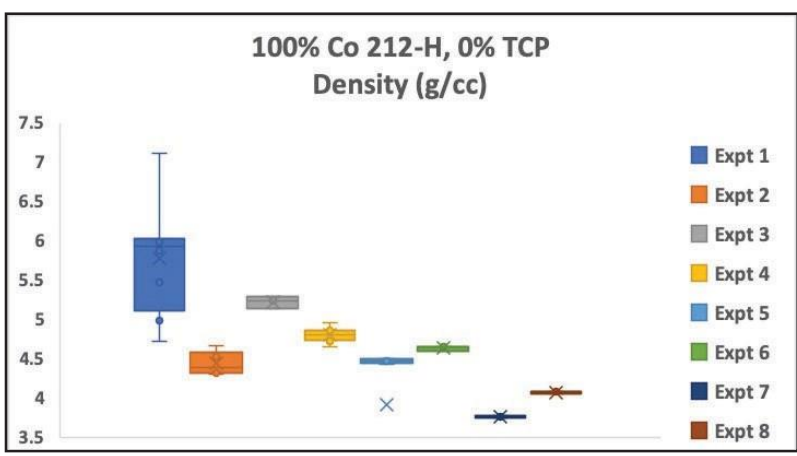

Fig. 5: Density variation for sample set 1 (100\% Co 212-H, 0\% TCP)
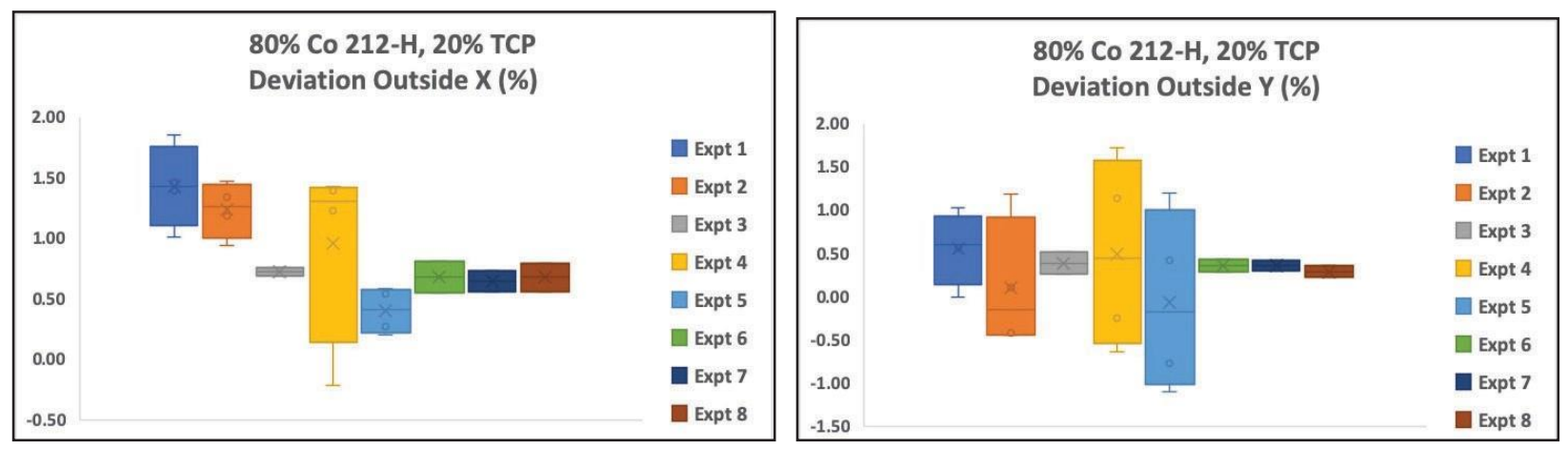


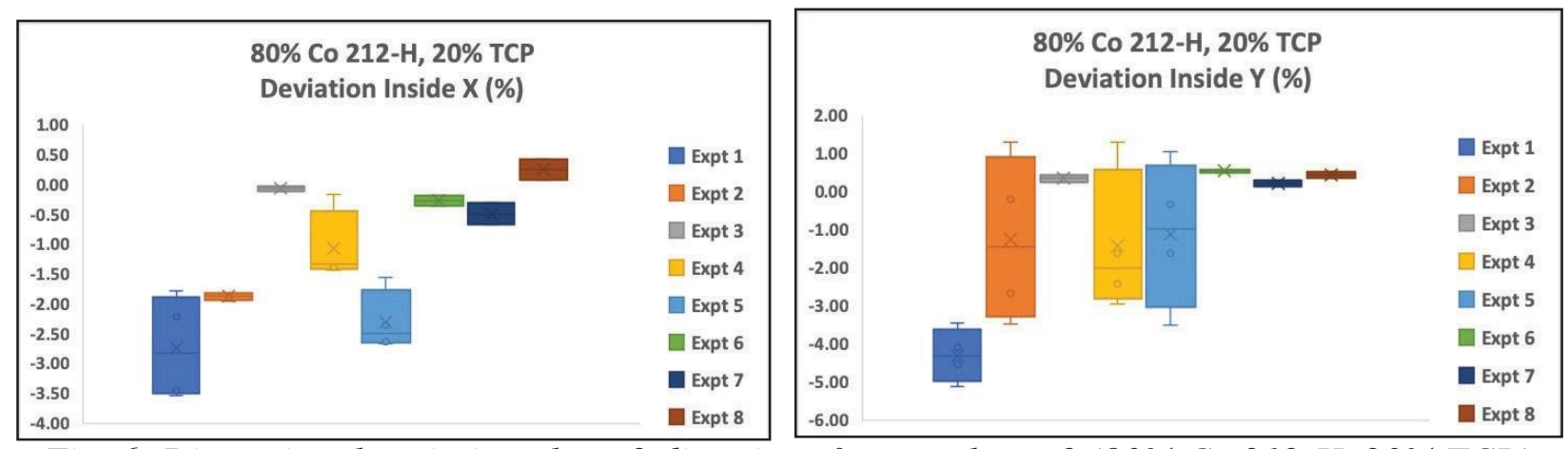

Fig. 6: Dimensional variation along 2 directions for sample set 2 (80\% Co 212-H, 20\% TCP)

The samples from various experiments were also photographed to look at the printing errors such as bleed out, distorted walls, rough surface, excess material etc. These are shown in Fig. 7-8.

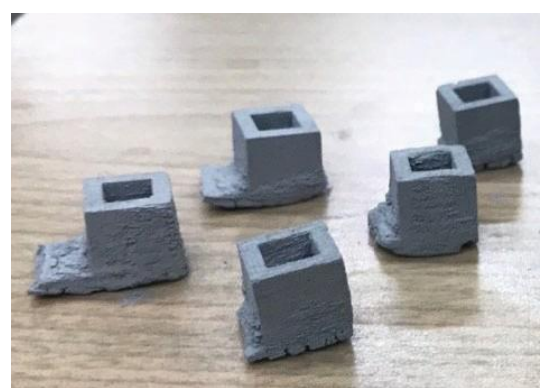

(a)

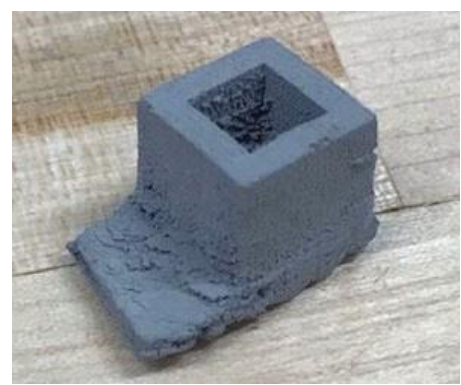

(b)

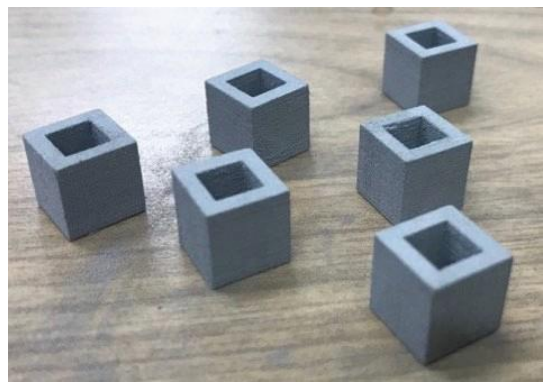

(c)

Fig. 7: (a) 10mm samples with printing bleed out, (b) 10mm sample with printing error $(c)$ Good 10mm samples

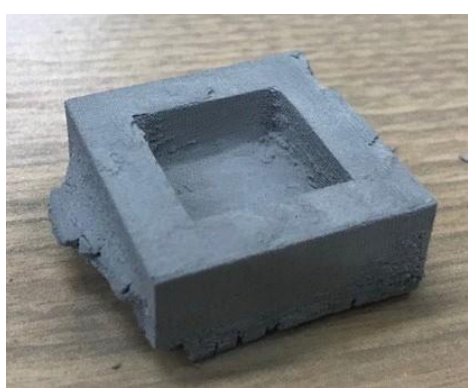

(a)

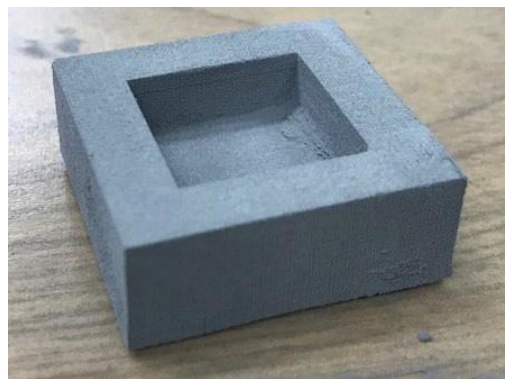

(b)

Fig. 8: (a) 30mm sample depicting printing error (b) Good 30mm sample

The response surface analysis of all the results was done using MINITAB. Only the main factors were considered in the analysis and the interaction between the factors was ignored for this study since the aim is to find the process parameters imports for dimensional variations. A typical fitted response surface is shown in Fig. 9. 


\section{O\% HA}

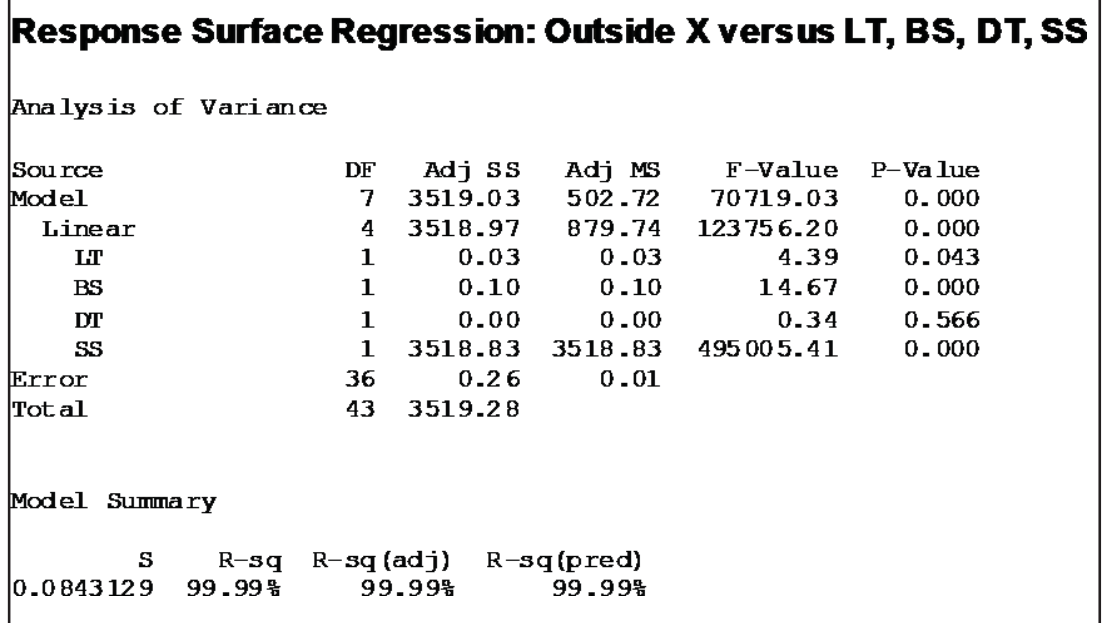

Fig. 9: Response surface result from MINITAB. LT: Layer Thickness, BS: Binder Saturation, DT: Drying time and SS: Sample Size

All the response surfaces were analyzed and the results of the P-values were summarized to identify the significant factors in each case. The summary of P-values is shown in Table 4 and 5.

\begin{tabular}{|c|c|c|c|c|}
\hline & Layer Thickness & Binder Saturation & Drying time & Sample Size \\
\hline Outside X & 0.043 & 0.001 & 0.566 & 0.001 \\
\hline Inside X & 0.107 & 0.901 & 0.250 & 0.001 \\
\hline Outside Y & 0.056 & 0.092 & 0.149 & 0.001 \\
\hline Inside Y & 0.140 & 0.932 & 0.585 & 0.001 \\
\hline Density & 0.001 & 0.013 & 0.814 & 0.001 \\
\hline
\end{tabular}

Table 4: P-Values for 100\% Co 212-H, 0\% TCP Samples

\begin{tabular}{|c|c|c|c|c|}
\hline & Layer Thickness & Binder Saturation & Drying time & Sample Size \\
\hline Outside X & 0.012 & 0.103 & 0.060 & 0.001 \\
\hline Inside X & 0.112 & 0.012 & 0.001 & 0.001 \\
\hline Outside Y & 0.066 & 0.099 & 0.093 & 0.001 \\
\hline Inside Y & 0.136 & 0.131 & 0.013 & 0.001 \\
\hline Density & 0.001 & 0.089 & 0.234 & 0.001 \\
\hline
\end{tabular}

Table 5: P-Values for $80 \%$ Co 212-H, $20 \%$ TCP Samples

\section{Discussion}

The study of various printing parameters and their effect on dimensions, surface finish and density of a novel biocomposite are studied in this work. Results from the various tests, the box plots and the response surface analysis show the following: 
a) There is a difference in the dimensional variations and density for both the $0 \%$ and $20 \%$ TCP samples.

b) Layer thickness and sample sizes are significant factors in both the sample sets. Thus, the dimension of the part, its surface and density are significantly affected by the layer thickness of the binder jet process. This is expected because the layer thickness affects the compaction of each layer and hence determines how much binder can saturate each powder layer.

c) Drying time is not a significant factor in $0 \%$ TCP samples but is important for the $20 \%$ TCP samples. The TCP is a much softer material than Co $212-\mathrm{H}$ and hence the binder saturates the powder a lot more in this case. This can result in material bleed outs or layers being dragged on another layer if it has not dried enough.

d) Similarly, binder saturation affects the dimensional accuracy more in case of $20 \%$ TCP samples than in the case of $0 \%$ TCP samples. In fact, in $0 \%$ TCP samples, binder saturation does not affect the inside dimensions. This is because once a layer is formed, the outside surface is more prone to the distortion or dislocation than the inside surface.

e) The $30 \mathrm{~mm}$ samples have much smaller variation in dimensions than the $10 \mathrm{~mm}$ samples. This might be because the larger surface area is more uniformly spread and dried than the smaller surface area of $10 \mathrm{~mm}$ samples.

f) By looking at the samples visually and their dimensions, Experiment 5 (60 $\mu \mathrm{m}$ Layer thickness, $80 \%$ binder saturation and $15 \mathrm{sec}$ drying time) performed the best for $0 \% \mathrm{TCP}$ Samples, 10mm size. In case of 20\% TCP samples, experiment 4 (80 $\mu \mathrm{m}$ Layer thickness, $80 \%$ binder saturation and $20 \mathrm{sec}$ drying time) performed the best.

g) There is a wide variation in the density of green parts depending upon the process parameters. This property can be taken advantage of to control the porosity of the parts made by various materials.

\section{Conclusions}

Traditional metals such as stainless steel, titanium and cobalt chrome are used in biomedical applications (implants, scaffolds etc.) but suffer from issues such as osseointegration and compatibility with existing bone. One way to improve traditional biomaterials is to incorporate ceramics with these metals so that their mechanical properties can be similar to cortical bones. TCP is such a ceramic with properties so that it can be used in human body. This research explores the use of binder jetting based additive manufacturing process to create a novel biocomposite made of cobalt chrome and tricalcium phosphate. Experiments were conducted and processing parameters were varied to study their effect on the printing of this biocomposite.

It is found that layer thickness is significant factor for printing with $0 \% \mathrm{TCP}$ and $20 \% \mathrm{TCP}$ samples. However, drying time is significant for 20\% TCP samples because of the soft nature of TCP powder. Similar effect is found with binder saturation. Thus, printing parameters are crucial in manufacturing parts from binder jet additive manufacturing. 
Further research needs to be conducted to take the best printing parameters and use the samples from this configuration and study the effect of sintering parameters on the strength and biocompatibility of these samples.

\section{References}

1. Tarafder, S., 2013. Physicomechanical, In Vitro and in Vivo Performance of 3D Printed Doped Tricalcium Phosphate Scaffolds for Bone Tissue Engineering and Drug Delivery, Ph.D. Dissertation,

2. Butscher, A., Bohner, M., Hofmann, S., Gauckler, L., Müller, R., 2011, Structural and material approaches to bone tissue engineering in powder-based three-dimensional printing, Acta Biomaterialia 7, pp. 907-920.

3. Gibson, I., Rosen, D. W., and Stucker, B., 2018, Additive Manufacturing Technologies: 3D Printing, Rapid Prototyping, and Direct Digital manufacturing, 2nd, Springer, New York, pp. 1-498.

4. Kolan, K., Thomas, A., Leu, M.C., Hilmas, G., 2018. In vitro assessment of laser sintered bioactive glass scaffolds with different pore geometries, Rapid Prototyping Journal, Vol. 21 Iss: 2, pp. $152-158$.

5. Bose, S., Vahabzadeh, S., Bandyopadhyay, A., 2018. Bone tissue engineering using 3D printing, Materials today, Volume 16, Issue 12, pp. 496-504.

6. Bose, S., Roy, M., Bandyopadhyay, A., 2018. Recent advances in bone tissue engineering scaffolds, Trends in Biotechnology, Volume 30, Issue 10, pp. 546-554.

7. Alvarez, K., Nakajima, H., 2009. Metallic Scaffolds for Bone Regeneration, Materials, 2, pp. 790-832; doi:10.3390/ma2030790.

8. MitraAsadi-Eydivand, MehranSolati-Hashjin, Farzad, A., 2016. Effect of technical parameters on porous structure and strength of 3D printed calcium sulfate prototypes, Robotics and Computer-Integrated Manufacturing, 37, pp. 57-67.

9. Cox, S.C., Thornby, J.A, Gibbons, G.J., Williams, M.A, Mallick, K.K, 2018. 3D printing of porous hydroxyapatite scaffolds intended for use in bone tissue engineering applications, Materials Science and Engineering C 47, pp. 237-247.

10. Ratner, B., Hoffman, A., Schoen, F., Lemons, J., 2012. Biomaterials Science : An introduction to materials in medicine, Academic Press, MA.

11. Lou, T., Wang, X., Song, G., Gu, Z., Yang, Z., 2015. Structure and properties of PLLA/b-TCP nanocomposite scaffolds for bone tissue engineering, J Mater Sci: Mater Med, pp. 26-34.

12. Ashby, M.F, Evans, A.G., Fleck, N.A., Gibson, N.A., Hutchinson, J.W., Wadley, H.N.G., 2000. Metal Foams: A Design Guide, Butterworth-Heinemann

13. German, R., 1996. Sintering Theory and Practice, Wiley-VCH

14. Bartolo, P., Kruth, J., Silva, J., Levy, G., Malshe, A., Rajurkar, K., Mitsuishi, M., Ciurana, J., Leu, M., "Biomedical production of implants by additive electro-chemical and physical processes”, CIRP Annals - Manufacturing Technology 61 (2018), 635-655

15. Hoath, S., 2016. "Fundamentals of Inkjet Printing: The science of inkjets and droplets", Wiley-VCH Verlag GmbH \& Co. 